\title{
Versatile Asymmetric Synthesis of the Kavalactones: The First Synthesis of (+)-Kavain
}

\author{
Thomas E. Smith, ${ }^{*}$ Mabel Djang, Alan J. Velander, C. Wade Downey, ${ }^{\dagger}$ Kathleen A. Carroll, and Sophie \\ van Alphen
}

Department of Chemistry, Williams College, Williamstown, Massachusetts 01267

\section{Supporting Information}

General Information. Oxygen- or moisture-sensitive reactions were carried out in flame-dried or ovendried glassware sealed with rubber septa under a positive pressure of dry nitrogen. Similarly sensitive liquids and solutions were transferred by gas-tight syringe or canula. Unless indicated otherwise, reagents and solvents were purchased and used without purification. Ether, THF, and $\mathrm{CH}_{2} \mathrm{Cl}_{2}, \mathrm{were}$ purified by passage through a bed of activated alumina. ${ }^{1}$ Analytical TLC was performed with $0.25 \mathrm{~mm}$ silica gel 60 plates with $254 \mathrm{~nm}$ fluorescent indicator from SiliCycle. Plates were visualized under UV light and treatment with either acidic $p$-anisaldehyde stain or aqueous ceric ammonium molybdate solution followed by gentle heating. The term flash chromatography refers to preparative silica gel column chromatography as described by Still and co-workers. ${ }^{2}$ Some chromatography was accomplished using an Isco Combiflash System Sg 100c. Silica gel 60, 230-240 mesh, was purchased from SiliCycle (R10030B). Analytical high performance liquid chromatography (HPLC) was carried out on an Agilent 1100 chromatograph equipped with a variable wavelength detector. ${ }^{1} \mathrm{H}$ NMR spectra were recorded on a Brüker Avance $500(500 \mathrm{MHz})$ or Brüker DPX300 (300 MHz) spectrometer and are reported in ppm using tetramethylsilane $(0.00 \mathrm{ppm})$ or solvent $\left(\mathrm{CDCl}_{3}: 7.24 \mathrm{ppm}\right)$ as an internal standard. Data are reported as $(\mathrm{ap}=$ apparent, $\mathrm{s}=$ singlet, $\mathrm{d}=$ doublet, $\mathrm{t}=$ triplet, $\mathrm{q}=$ quartet, $\mathrm{m}=$ multiplet, $\mathrm{b}=$ broad; coupling constant(s) in $\mathrm{Hz}$; integration. Proton-decoupled ${ }^{13} \mathrm{C}$ NMR spectra were recorded at $125 \mathrm{MHz}$ or $75 \mathrm{MHz}$ and are reported in ppm using solvent as an internal standard $\left(\mathrm{CDCl}_{3}\right.$ : $77.00 \mathrm{ppm}$ ). Infrared spectra were recorded as thin films on $\mathrm{NaCl}$ plates on a Perkin-Elmer 710 Series Fourier transform spectrometer (FTIR). Melting points were determined with a Laboratory Devices MelTemp II apparatus equipped with an Fluke Model $51 \mathrm{~K} / \mathrm{J}$ thermocouple and are uncorrected. Optical rotations were measured on a Perkin-Elmer 241 digital polarimeter using the sodium (589, D line) lamp and are reported as follows: $[\alpha]_{\lambda}{ }^{\mathrm{T}}{ }^{\circ} \mathrm{C}(\mathrm{c}=\mathrm{g} / 100 \mathrm{~mL}$, solvent). Combustion analyses were performed by Atlantic Microlab, Norcross, Georgia. High Resolution mass spectra (HRMS) were recorded at the Nebraska Center for Mass Spectrometry.

General Procedure: $\mathrm{TiCl}_{4}$-mediated aldol reaction of $\boldsymbol{N}$-acetylthiazolidinethione with aldehydes. ${ }^{3}$ To a $25 \mathrm{~mL}$ round-bottom flask was added $N$-acetyl thiazolidinethione 7 (2.5 mmol, 1.0 equiv) in 12.6 $\mathrm{mL} \mathrm{CH}_{2} \mathrm{Cl}_{2}(0.2 \mathrm{M}$ in 7$)$. The bright yellow solution was cooled to $-78{ }^{\circ} \mathrm{C}, \mathrm{TiCl}_{4}(2.75 \mathrm{mmol}, 1.1$ equiv) was added, and the suspension was warmed to $-40{ }^{\circ} \mathrm{C}$. After $10 \mathrm{~min}$, the resulting homogeneous orange solution was recooled to $-78{ }^{\circ} \mathrm{C}$ and ${ }^{i} \operatorname{Pr}_{2} \mathrm{NEt}$ ( $2.75 \mathrm{mmol}, 1.1$ equiv) was added. The blood-red solution was warmed to $-40{ }^{\circ} \mathrm{C}$ and stirred for $2 \mathrm{~h}$. This titanium enolate solution was recooled to $-78{ }^{\circ} \mathrm{C}$ and the aldehyde ( $2.75 \mathrm{mmol}, 1.1$ equiv) was added dropwise with rapid stirring. After $1 \mathrm{~h}$, the reaction was quenched by pouring into $25 \mathrm{~mL}$ of rapidly stirring sat. aq. $\mathrm{NH}_{4} \mathrm{Cl}$. The layers were separated and the aqueous portion was extracted with $\mathrm{CH}_{2} \mathrm{Cl}_{2}(3 \times 5 \mathrm{~mL})$. The combined organic layers were dried over

(1) Pangborn, A. B.; Giardello, M. A.; Grubbs, R. H.; Rosen, R. K.; Timmers, F. J. Organometallics 1996, 1518.

(2)Still, W. C.; Kahn, M.; Mitra, A. J. Org. Chem 1978, 43, 2923.

(3) Adapted from the general procedure of González, Á.; Aiguadé, J.; Urpí, F.; Vilarrasa, J. Tetrahedron Lett. 1996, $37,8949$. 
$\mathrm{Na}_{2} \mathrm{SO}_{4}$ and concentrated in vacuo. The diastereomeric ratio of the crude product was determined by NMR and HPLC (Zorbax sil. Column, 20\% EtOAc/hexanes, $2.5 \mathrm{~mL} / \mathrm{min}, 305 \mathrm{~nm}$ ). Flash chromatography (0-50\% $\mathrm{Et}_{2} \mathrm{O} /$ hexanes) afforded the pure major diastereomer.

\section{$(E)(R)-3-h y d r o x y-1-((S)-4-i s o p r o p y l-2-t h i o x o t h i a z o l i d i n-3-y l)-5-p h e n y l p e n t-4-e n-1-o n e ~(8 a){ }^{4}$}
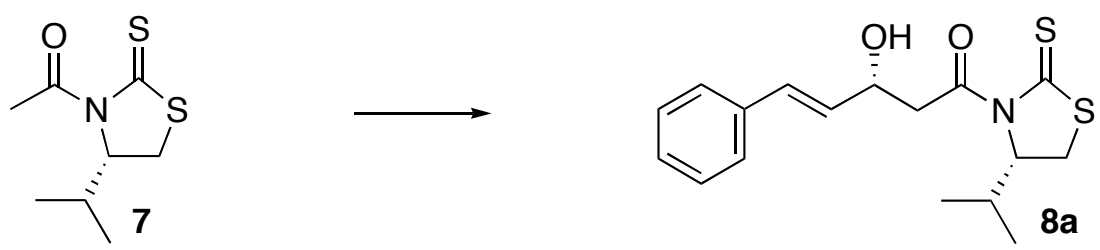

The title compound was prepared according to General Procedure (thiazolidinethione 7, $511 \mathrm{mg}, 2.51$ mmol; $\mathrm{TiCl}_{4}, 304 \mu \mathrm{L}, 2.76 \mathrm{mmol} ;{ }^{i} \mathrm{Pr}_{2} \mathrm{NEt}, 482 \mu \mathrm{L}, 2.76 \mathrm{mmol}$, cinnamaldehyde, $\left.349 \mu \mathrm{L}, 2.76 \mathrm{mmol}\right)$ and isolated as a bright yellow oil $\left(710 \mathrm{mg}, 84 \%\right.$ yield): ${ }^{1} \mathrm{H} \mathrm{NMR}\left(300 \mathrm{MHz}, \mathrm{CDCl}_{3}\right) \delta 7.41-7.19$ (m, $5 \mathrm{H}), 6.67(\mathrm{~d}, 1 \mathrm{H}, \mathrm{J}=16.0 \mathrm{~Hz}), 6.27(\mathrm{dd}, 1 \mathrm{H}, \mathrm{J}=15.9,5.9 \mathrm{~Hz}), 5.15(\mathrm{dd}, 1 \mathrm{H}, \mathrm{J}=7.3,6.8 \mathrm{~Hz}), 4.90-4.80$ $(\mathrm{m}, 1 \mathrm{H}), 3.73(\mathrm{dd}, 1 \mathrm{H}, \mathrm{J}=17.5,3.1 \mathrm{~Hz}), 3.49(\mathrm{dd}, 1 \mathrm{H}, \mathrm{J}=11.5,7.9 \mathrm{~Hz}), 3.43(\mathrm{dd}, 1 \mathrm{H}, \mathrm{J}=17.5,8.7 \mathrm{~Hz})$, 3.06 (b s, 1H), $3.00(\mathrm{~d}, 1 \mathrm{H}, \mathrm{J}=11.5 \mathrm{~Hz}), 2.42-2.30(\mathrm{~m}, 1 \mathrm{H}), 1.05(\mathrm{~d}, 3 \mathrm{H}, \mathrm{J}=6.8 \mathrm{~Hz}), 0.98(\mathrm{~d}, 3 \mathrm{H}, \mathrm{J}=$ $7.0 \mathrm{~Hz}) \mathrm{ppm} ;{ }^{13} \mathrm{C} \mathrm{NMR}\left(75 \mathrm{MHz}, \mathrm{CDCl}_{3}\right) \delta$ 203.0, 172.3, 136.4, 130.5, 130.0, 128.5, 127.7, 126.5, 71.3, 68.7, 45.2, 30.8, 30.6, 19.0, 17.1 ppm; IR (film) 3423, 2963, 1691, 1467, 1363, 1312, 1257, 1164, 1093, $1041,968,750,693 \mathrm{~cm}^{-1} ;[\alpha]_{\mathrm{D}}{ }^{25}=+310.5^{\circ}\left(c=1.41, \mathrm{CHCl}_{3}\right)$; HRMS $(\mathrm{CI})$ : Exact mass calcd for $\mathrm{C}_{17} \mathrm{H}_{21} \mathrm{NO}_{2} \mathrm{~S}_{2} \mathrm{Li}[\mathrm{M}+\mathrm{Li}]^{+}:$342.1174; Found: 342.1174; Anal Calcd for $\mathrm{C}_{17} \mathrm{H}_{21} \mathrm{NO}_{2} \mathrm{~S}_{2}: \mathrm{C}, 60.86 \%, \mathrm{H}$, $6.31 \%, \mathrm{~N}, 4.18 \%$; Found: C, 60.61\%, H, 6.49\%, N, $4.11 \%$.

\section{(S)-3-hydroxy-1-((S)-4-isopropyl-2-thioxothiazolidin-3-yl)-5-phenylpentan-1-one (8b)}
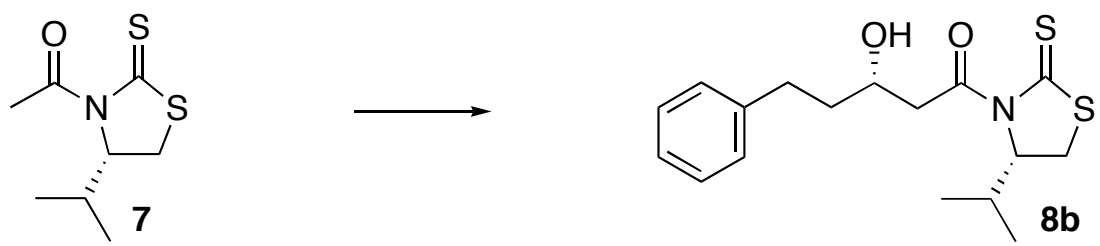

The title compound was prepared according to General Procedure (thiazolidinethione 7, $279 \mathrm{mg}, 1.37$ mmol, 1.2 equiv; $\mathrm{TiCl}_{4}, 181 \mu \mathrm{L}, 1.65 \mathrm{mmol}, 1.44$ equiv; ${ }^{i} \operatorname{Pr}_{2} \mathrm{NEt}, 288 \mu \mathrm{L}, 1.65 \mathrm{mmol}, 1.44$ equiv, dihydrocinnamaldehyde, $151 \mu \mathrm{L}, 1.14 \mathrm{mmol}, 1$ equiv) and isolated as bright yellow crystals (201 mg, 54\% yield): ${ }^{1} \mathrm{H}$ NMR (300 MHz, $\left.\mathrm{CDCl}_{3}\right) \delta$ 7.31-7.14 (m, 5H), 5.14 (dd, $\left.1 \mathrm{H}, \mathrm{J}=7.3,6.7 \mathrm{~Hz}\right), 4.19-4.08$ $(\mathrm{m}, 1 \mathrm{H}), 3.63(\mathrm{dd}, 1 \mathrm{H}, \mathrm{J}=17.8,2.5 \mathrm{~Hz}), 3.49(\mathrm{dd}, 1 \mathrm{H}, \mathrm{J}=11.5,8.0 \mathrm{~Hz}), 3.17(\mathrm{dd}, 1 \mathrm{H}, \mathrm{J}=17.8,9.2 \mathrm{~Hz})$, $3.00(\mathrm{~d}, 1 \mathrm{H}, \mathrm{J}=11.4 \mathrm{~Hz}), 2.98$ (b s, 1H), 2.89-2.78 (m, 1H), 2.77-2.65 (m, 1H), 2.40-2.27 (m, 1H), 1.96$1.72(\mathrm{~m}, 2 \mathrm{H}), 1.05(\mathrm{~d}, 3 \mathrm{H}, \mathrm{J}=6.8 \mathrm{~Hz}), 0.96(\mathrm{~d}, 3 \mathrm{H}, \mathrm{J}=6.9 \mathrm{~Hz}) \mathrm{ppm} ;{ }^{13} \mathrm{C}$ NMR $\left(75 \mathrm{MHz}, \mathrm{CDCl}_{3}\right)$ ठ 202.9, 173.0, 141.7, 128.4, 128.3, 125.7, 71.2, 67.1, 45.5, 37.8, 31.7, 30.7, 30.4, 19.0, 17.7 ppm; IR (KCl wafer) $3568,3535,2960,1682,1365,1242,1162,1092,1042,702 \mathrm{~cm}^{-1} ;$ m.p. $=96-98{ }^{\circ} \mathrm{C} ;[\alpha]_{\mathrm{D}}{ }^{23}=$ $+351^{\circ}\left(c=1.00, \mathrm{CH}_{2} \mathrm{Cl}_{2}\right)$; HRMS (EI): Exact mass calcd for $\mathrm{C}_{17} \mathrm{H}_{23} \mathrm{NO}_{2} \mathrm{~S}_{2}[\mathrm{M}]^{+}: 337.1170$; Found: 337.1158; Anal calcd for $\mathrm{C}_{17} \mathrm{H}_{23} \mathrm{NO}_{2} \mathrm{~S}_{2:}$ : C, 60.50\%, H, 6.87\%, N, 4.15\%; Found: C, 60.59\%, H, 6.77\%, $\mathrm{N}, 4.21 \%$.

(4) Jeong, T. M.; Park, K. H. J. Korean Chem. Soc. 1989, 33, 426. See also reference 3. 


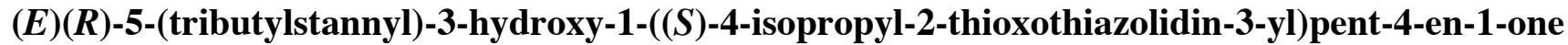

(8c)
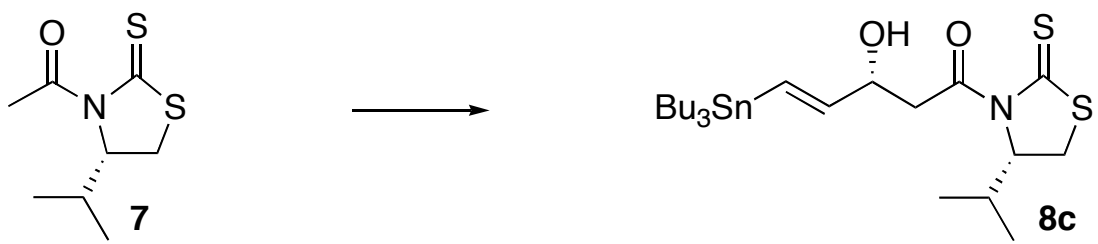

To a $25 \mathrm{~mL}$ round-bottom flask in a dry box was added $\mathrm{Sn}(\mathrm{OTf})_{2}(450 \mathrm{mg}, 1.08 \mathrm{mmol}, 2.3$ equiv, Strem Chemicals). The flask was fitted with a rubber septum and was moved to the hood. After attaching an argon line, $\mathrm{CH}_{2} \mathrm{Cl}_{2}(3.0 \mathrm{~mL})$ was added. The gray suspension was cooled to $-50{ }^{\circ} \mathrm{C}$ and $\mathrm{N}$ ethylpiperidine (148 $\mu \mathrm{L}, 1.08 \mathrm{mmol}, 2.3$ equiv) was added. To the resulting light yellow solution was added thiazolidinethione 7 (143 mg, $0.704 \mathrm{mmol}, 1.5$ equiv) in $\mathrm{CH}_{2} \mathrm{Cl}_{2}$ (2.1 mL). After stirring for $4 \mathrm{~h}$ at $-50{ }^{\circ} \mathrm{C}$, the tin enolate solution was cooled to $-78{ }^{\circ} \mathrm{C}$ and $(E)-3$-(tributylstannyl)-2-propenal ${ }^{5}$ (162 $\mathrm{mg}$, $0.469 \mathrm{mmol}, 1.0$ equiv) was added in $\mathrm{CH}_{2} \mathrm{Cl}_{2}(2.5 \mathrm{~mL})$ over $10 \mathrm{~min}$. After $30 \mathrm{~min}$, the reaction was quenched pouring into $\mathrm{pH} 7$ buffer $(7.0 \mathrm{~mL})$ with rapid stirring. The subsequent emulsion was filtered through Celite, sat. aq. $\mathrm{NH}_{4} \mathrm{Cl}(25 \mathrm{~mL})$ was added, and the layers were separated. The aqueous layer was extracted with $\mathrm{CH}_{2} \mathrm{Cl}_{2}(3 \times 25 \mathrm{~mL})$ and the combined organic layers were dried over $\mathrm{Na}_{2} \mathrm{SO}_{4}$. Filtration and concentrated in vacuo afforded a greenish-yellow oil. Flash chromatography on Silicycle $\mathrm{Si}$-Diol functionalized silica gel (0-50\% $\mathrm{Et}_{2} \mathrm{O} /$ hexanes) provided the title compound as an orange oil (213 $\mathrm{mg}$, 83\%): ${ }^{1} \mathrm{H}$ NMR $\left(300 \mathrm{MHz}, \mathrm{CDCl}_{3}\right) \delta 6.26(\mathrm{dd}, 1 \mathrm{H}, \mathrm{J}=19.2,1.2 \mathrm{~Hz}), 6.06(\mathrm{dd}, 1 \mathrm{H}, \mathrm{J}=19.2,4.8), 5.15$ $(\mathrm{dd}, 1 \mathrm{H}, \mathrm{J}=7.4,6.7 \mathrm{~Hz}), 4.69-4.60(\mathrm{~m}, 1 \mathrm{H}), 3.65(\mathrm{dd}, 1 \mathrm{H}, \mathrm{J}=17.5,3.0 \mathrm{~Hz}), 3.52(\mathrm{dd}, 1 \mathrm{H}, \mathrm{J}=11.5,7.9$ $\mathrm{Hz}), 3.32(\mathrm{dd}, 1 \mathrm{H}, \mathrm{J}=17.5,8.9 \mathrm{~Hz}), 3.04(\mathrm{dd}, 1 \mathrm{H}, \mathrm{J}=11.5,0.8 \mathrm{~Hz}) 2.86(\mathrm{~b} \mathrm{~s}, 1 \mathrm{H}), 2.45-2.31(\mathrm{~m}, 1 \mathrm{H})$, 1.56-1.43 (m, 6H), 1.38-1.22 (m, 6H), $1.07(\mathrm{~d}, 3 \mathrm{H}, \mathrm{J}=6.8 \mathrm{~Hz}), 1.01(\mathrm{~d}, 3 \mathrm{H}, \mathrm{J}=7.6 \mathrm{~Hz}), 0.94-0.84(\mathrm{~m}$, $15 \mathrm{H}) \mathrm{ppm} ;{ }^{13} \mathrm{C} \mathrm{NMR}\left(75 \mathrm{MHz}, \mathrm{CDCl}_{3}\right) \delta 202.9,172.6,148.1,128.8,71.4,70.9,45.1,30.8,30.7,29.0$, 27.2, 19.0, 17.8, 13.7, 9.4 ppm ; IR (film) 3417, 2957, 2926, 1695, 1464, 1374, 1255, 1167, 1043, 989, $865,662 \mathrm{~cm}^{-1} ;[\alpha]_{\mathrm{D}}{ }^{21.5}=+219.9^{\circ}\left(c=1.00, \mathrm{CH}_{2} \mathrm{Cl}_{2}\right)$; Anal calcd for $\mathrm{C}_{23} \mathrm{H}_{43} \mathrm{NO}_{2} \mathrm{~S}_{2} \mathrm{Sn}: \mathrm{C}, 50.37 \%, \mathrm{H}$, $7.90 \%$, N, 2.55\%; Found: C, 50.64\%, H, 8.02\%, N, $2.59 \%$.

General Procedure: Transacylation of thiazolidinethione aldol adducts. To a $10 \mathrm{~mL}$ concentration flask containing a solution of thiazolidinethione aldol adduct $(0.346 \mathrm{mmol}, 1$ equiv) in THF $(0.86 \mathrm{~mL}$, $0.4 \mathrm{M}$ ) was added potassium ethyl malonate $\left(0.692 \mathrm{mmol}, 2.0\right.$ equiv, Aldrich) followed by $\mathrm{MgCl}_{2}(0.346$ mmol, 1.0 equiv, Aldrich \#24,413-9). The flask was flushed with Ar and the suspension was stirred rapidly at $\mathrm{rt}$. After $30 \mathrm{~min}$, imidazole $(0.346 \mathrm{mmol}, 1.0$ equiv) was added in one portion and the suspension stirred rapidly at rt overnight. During this time the bright yellow color slowly faded to a very light yellow. The reaction was diluted with EtOAc $(10 \mathrm{~mL})$, washed with $0.5 \mathrm{M} \mathrm{HCl}(5 \mathrm{~mL})$, and the aqueous layer was back-extracted with EtOAc $(3 \times 2 \mathrm{~mL})$. The combined organic layers were then washed with $0.5 \mathrm{M} \mathrm{NaHCO}_{3}(5 \mathrm{~mL})$ and back-extracted with EtOAc $(3 \times 2 \mathrm{~mL})$. Finally, the combined organic layers were dried over $\mathrm{Na}_{2} \mathrm{SO}_{4}$, filtered, and concentrated in vacuo to give a light yellow oil containing the desired product contaminated with free thiazolidinethione auxiliary. Flash chromatography $\left(0 \rightarrow 30 \%\right.$ EtOAc/hexanes or $\left.0 \rightarrow 8 \% \mathrm{Et}_{2} \mathrm{O} / \mathrm{CH}_{2} \mathrm{Cl}_{2}\right)$ afforded the pure $\delta$-hydroxy- $\beta$ ketoester.

(5) Oswald, R.; Chavant, P.; Stadtmüller, H.; Knochel, P. J. Org. Chem. 1994, 59, 4143. 


\section{Ethyl $(E)(R)-5$-hydroxy-3-oxo-7-phenylhept-6-enoate (9a)}

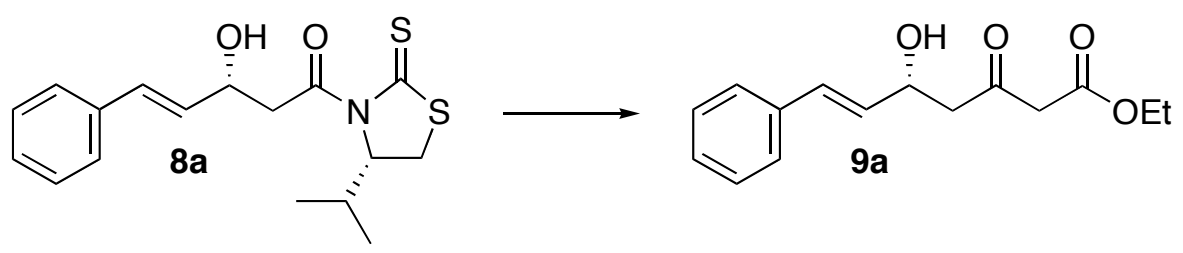

The title compound was prepared according to General Procedure (thiazolidinethione 8a, $116 \mathrm{mg}, 0.346$ mmol; potassium ethyl malonate, $118 \mathrm{mg}, 0.692 \mathrm{mmol} ; \mathrm{MgCl}_{2}, 33 \mathrm{mg}, 0.346 \mathrm{mmol}$; imidazole, $24 \mathrm{mg}$, $0.346 \mathrm{mmol}$; stirred for $7 \mathrm{~h}$ ) and isolated as a pale yellow oil ( $80 \mathrm{mg}, 88 \%):{ }^{1} \mathrm{H}$ NMR (300 $\mathrm{MHz}, \mathrm{CDCl}_{3}$ ) ठ 7.44-7.21 (m, 5H), $6.65(\mathrm{~d}, 1 \mathrm{H}, \mathrm{J}=15.9 \mathrm{~Hz}), 6.21(\mathrm{dd}, 1 \mathrm{H}, \mathrm{J}=15.9,6.1 \mathrm{~Hz}), 4.84-4.75(\mathrm{~m}, 1 \mathrm{H}), 4.20$ $(\mathrm{q}, 2 \mathrm{H}, \mathrm{J}=7.1 \mathrm{~Hz}), 3.51(\mathrm{~s}, 2 \mathrm{H}), 2.87(\mathrm{~d}, 2 \mathrm{H}, \mathrm{J}=6.0 \mathrm{~Hz}), 1.28(\mathrm{t}, 3 \mathrm{H}, \mathrm{J}=7.1 \mathrm{~Hz}) \mathrm{ppm} ;{ }^{13} \mathrm{C}$ NMR $(75$ $\mathrm{MHz}, \mathrm{CDCl}_{3}$ ) $\delta$ 202.2, 166.4, 135.9, 130.2, 129.3, 128.1, 127.3, 126.0, 67.9, 61.1, 49.5, 49.1, 13.6 ppm; IR (film) 3467, 2982, 1734, 1713, 1033, 972, 748, $693 \mathrm{~cm}^{-1} ;[\alpha]_{\mathrm{D}}^{19.2}=+14.0^{\circ}\left(c=1.00, \mathrm{CH}_{2} \mathrm{Cl}_{2}\right)$; HRMS (CI): Exact mass calcd for $\mathrm{C}_{15} \mathrm{H}_{18} \mathrm{O}_{4} \mathrm{Li}[\mathrm{M}+\mathrm{Li}]^{+}: 269.1365$; Found: 269.1378; Anal calcd for $\mathrm{C}_{15} \mathrm{H}_{18} \mathrm{O}_{4}$ : C, 68.68\%, H, 6.92\%; Found: C, 68.93\%, H, 6.79\%.

\section{Ethyl $(S)-5-h y d r o x y-3-o x o-7-p h e n y l h e p t a n o a t e(9 b)$}

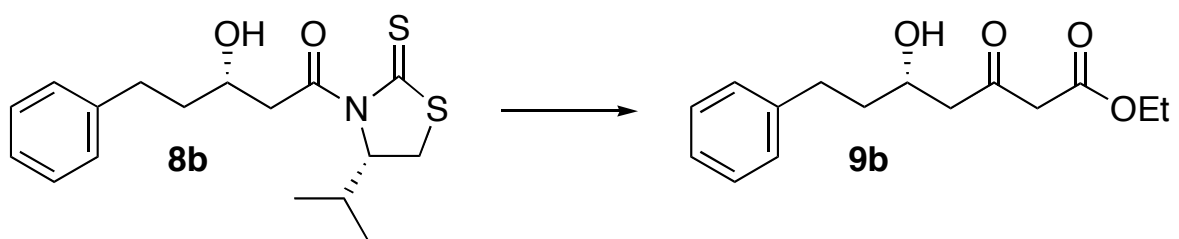

The title compound was prepared according to General Procedure (thiazolidinethione $\mathbf{8 b}, 99 \mathrm{mg}, 0.29$ mmol; potassium ethyl malonate, $100 \mathrm{mg}, 0.59 \mathrm{mmol} ; \mathrm{MgCl}_{2}, 28 \mathrm{mg}, 0.29 \mathrm{mmol}$; imidazole, $20 \mathrm{mg}$, $0.29 \mathrm{mmol}$; stirred for $6 \mathrm{~h})$ and isolated as a pale yellow oil $(68 \mathrm{mg}, 89 \%):{ }^{1} \mathrm{H} \mathrm{NMR}\left(300 \mathrm{MHz}, \mathrm{CDCl}_{3}\right)$ $\delta$ 7.31-7.12 (m, 5H), $2.68(\mathrm{q}, 2 \mathrm{H}, \mathrm{J}=7.1 \mathrm{~Hz}), 4.11-4.03(\mathrm{~m}, 1 \mathrm{H}), 3.44(\mathrm{~s}, 2 \mathrm{H}), 2.96(\mathrm{~d}, 1 \mathrm{H}, \mathrm{J}=3.8 \mathrm{~Hz})$, 2.86-2.61 (m, 4H), 1.89-1.64 (m, 2H), $1.26(\mathrm{t}, 3 \mathrm{H}, \mathrm{J}=7.1 \mathrm{~Hz}) \mathrm{ppm} ;{ }^{13} \mathrm{C} \mathrm{NMR}\left(75 \mathrm{MHz}, \mathrm{CDCl}_{3}\right) \delta 203.5$, 166.8, 141.6, 128.3 (2 peaks), 125.8, 66.7, 61.4, 49.8, 49.6, 38.0, 31.6, 14.0 ppm ; IR (film) 3442, 3027, $2931,1741,1712,1496,1455,1409,1368,1318,1155,1029,750,701 \mathrm{~cm}^{-1} ;[\alpha]_{D}^{23}=+13^{\circ}(c=1.00$, $\mathrm{CH}_{2} \mathrm{Cl}_{2}$ ); HRMS (EI): Exact mass calcd for $\mathrm{C}_{15} \mathrm{H}_{18} \mathrm{O}_{3}$ [M $\left.-\mathrm{H}_{2} \mathrm{O}\right]^{+}$: 246.1256; Found: 246.1265; Anal calcd for $\mathrm{C}_{15} \mathrm{H}_{20} \mathrm{O}_{4}$ : C, 68.16\%, H, 7.63\%; Found: C, 67.88\%, H, 7.72\%.

\section{Ethyl $(E)(R)-7-($ tributylstannyl)-5-hydroxy-3-oxohept-6-enoate (9c)}

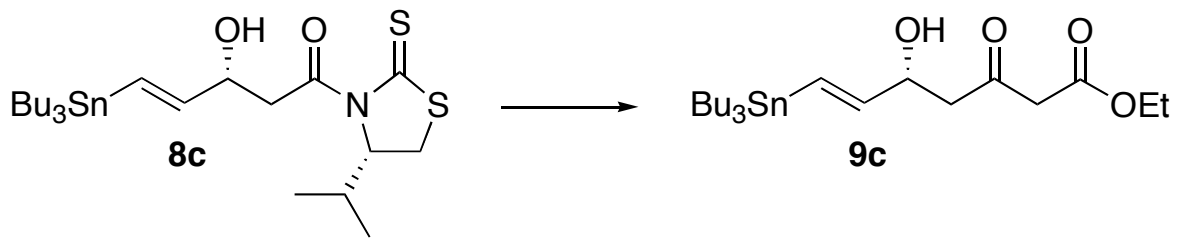

The title compound was prepared according to General Procedure (thiazolidinethione 8c, $3.374 \mathrm{~g}, 6.14$ mmol; potassium ethyl malonate, $2.091 \mathrm{~g}, 12.3 \mathrm{mmol} ; \mathrm{MgCl}_{2}, 585 \mathrm{mg}, 6.14 \mathrm{mmol}$; imidazole, $418 \mathrm{mg}$, $6.14 \mathrm{mmol}$; stirred overnight) and isolated as a yellow oil $(2.395 \mathrm{~g}, 82 \%):{ }^{1} \mathrm{H} \mathrm{NMR}\left(500 \mathrm{MHz}, \mathrm{CDCl}_{3}\right)$ $\delta 6.23(\mathrm{dd}, 1 \mathrm{H}, \mathrm{J}=19.1,1.3 \mathrm{~Hz}), 6.00(\mathrm{dd}, 1 \mathrm{H}, \mathrm{J}=19.1,5.0 \mathrm{~Hz}), 4.60-4.54(\mathrm{~m}, 1 \mathrm{H}), 4.21(\mathrm{q}, 2 \mathrm{H}, \mathrm{J}=7.2$ 
$\mathrm{Hz}), 3.49$ (s, 2H), 2.82-2.72 (m, 2H), $2.68(\mathrm{bs} 1 \mathrm{H}), 1.57-1.40(\mathrm{~m}, 6 \mathrm{H}), 1.37-1.23(\mathrm{~m}, 9 \mathrm{H}), 0.95-0.84(\mathrm{~m}$, 15H) ppm; ${ }^{13} \mathrm{C}$ NMR (125 MHz, $\left.\mathrm{CDCl}_{3}\right) \delta 202.8,166.9,148.1,128.9,70.7,61.5,50.0,49.4,29.0,27.2$, 14.1, 13.6, 9.4 ppm ; IR (film) 3453, 2957, 2926, 2853, 1742, 1714, 1652, 1464, 1376, 1316, 1235, $1177,1033,874,690,666,596,507 \mathrm{~cm}^{-1} ;[\alpha]_{\mathrm{D}}{ }^{21}=+13.8^{\circ}\left(c=1.00, \mathrm{CH}_{2} \mathrm{Cl}_{2}\right)$.

\section{Ethyl $(E)(4 R, 5 S)-5-h y d r o x y-4-m e t h y l-3-o x o-7-p h e n y l h e p t-6-e n o a t e ~(23)$}

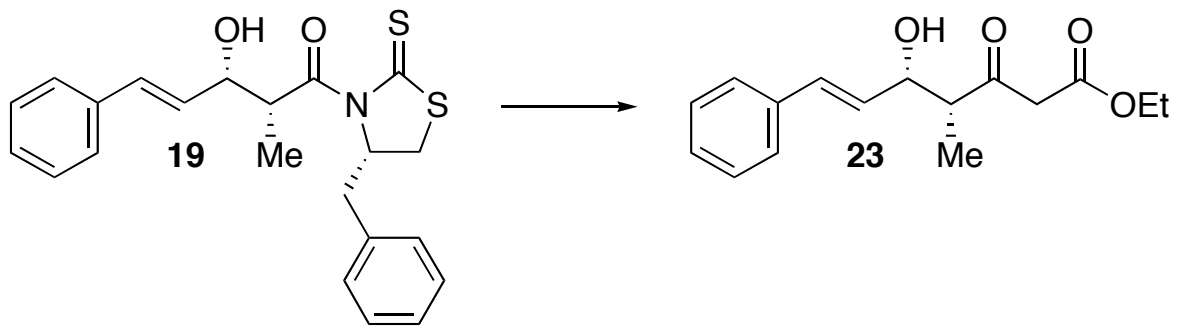

The title compound was prepared according to General Procedure (thiazolidinethione 19, $226 \mathrm{mg}, 0.569$ mmol; potassium ethyl malonate, $194 \mathrm{mg}, 1.137 \mathrm{mmol} ; \mathrm{MgCl}_{2}, 54 \mathrm{mg}, 0.569 \mathrm{mmol}$; imidazole, $39 \mathrm{mg}$, $0.569 \mathrm{mmol}$; stirred overnight) and isolated as a pale yellow oil $(94.0 \mathrm{mg}, 60 \%):{ }^{1} \mathrm{H} \mathrm{NMR}(500 \mathrm{MHz}$, $\left.\mathrm{CDCl}_{3}\right) \delta$ 7.44-7.18 (m, 5H), $6.64(\mathrm{~d}, 1 \mathrm{H}, \mathrm{J}=15.9 \mathrm{~Hz}), 6.16(\mathrm{dd}, 1 \mathrm{H}, \mathrm{J}=15.9,6.1 \mathrm{~Hz}), 4.71-4.60(\mathrm{~m}$, $1 \mathrm{H}), 4.19(\mathrm{q}, 2 \mathrm{H}, \mathrm{J}=7.1 \mathrm{~Hz}), 3.63-3.53(\mathrm{~m}, 2 \mathrm{H}), 3.00-2.90(\mathrm{~m}, 1 \mathrm{H}), 2.61(\mathrm{bs}, 1 \mathrm{H}), 1.27(\mathrm{t}, 3 \mathrm{H}, \mathrm{J}=7.1$ $\mathrm{Hz}), 1.20(\mathrm{~d}, 3 \mathrm{H}, \mathrm{J}=7.2 \mathrm{~Hz}) \mathrm{ppm} ;{ }^{13} \mathrm{C} \mathrm{NMR}\left(125 \mathrm{MHz}, \mathrm{CDCl}_{3}\right) \delta$ 206.5, 167.2, 136.4, 131.7, 128.6, $128.5,127.8,126.5,72.5,61.5,51.3,48.8,14.1,10.6$ ppm ; IR (film) 3500, 2981, 2926, 1739, 1709, $1646,1450,1369,1313,1258,1157,1022,969,753,696 \mathrm{~cm}^{-1} ;[\alpha]_{\mathrm{D}}^{21.5}=-18.2^{\circ}\left(c=1.00, \mathrm{CH}_{2} \mathrm{Cl}_{2}\right)$.

\section{Ethyl $(E)(4 S, 5 R)-5-h y d r o x y-4-m e t h y l-3-o x o-7-p h e n y l h e p t-6-e n o a t e ~(24)$}

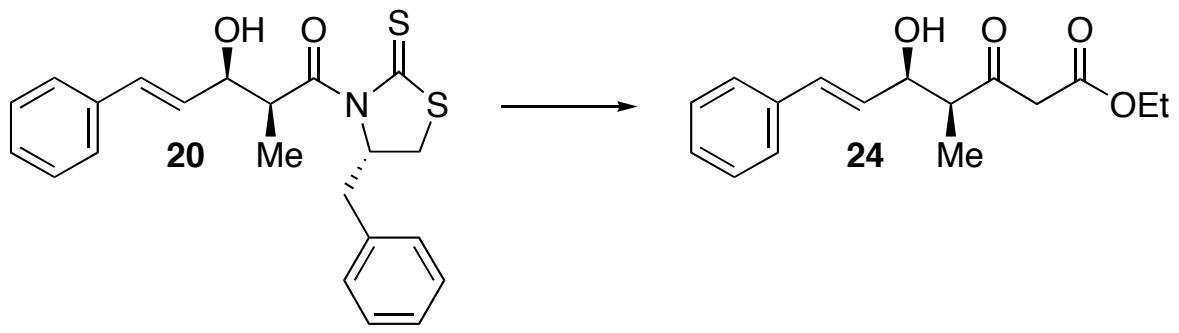

The title compound was prepared according to General Procedure (thiazolidinethione 20, $74 \mathrm{mg}, 0.186$ mmol; potassium ethyl malonate, $63 \mathrm{mg}, 0.372 \mathrm{mmol} ; \mathrm{MgCl}_{2}, 18 \mathrm{mg}, 0.186 \mathrm{mmol}$; imidazole, $13 \mathrm{mg}$, $0.186 \mathrm{mmol}$; stirred overnight) and isolated as a pale yellow oil $(30.7 \mathrm{mg}, 60 \%)$. All spectral data were identical to those reported for $\mathbf{2 3}$ above except for the optical rotation: $[\alpha]_{\mathrm{D}}^{21.5}=+16.2^{\circ}(c=1.00$, $\mathrm{CH}_{2} \mathrm{Cl}_{2}$; 


\section{Ethyl $(E)(4 S, 5 S)-5-h y d r o x y-4-m e t h y l-3-o x o-7-p h e n y l h e p t-6-e n o a t e ~(25)$}

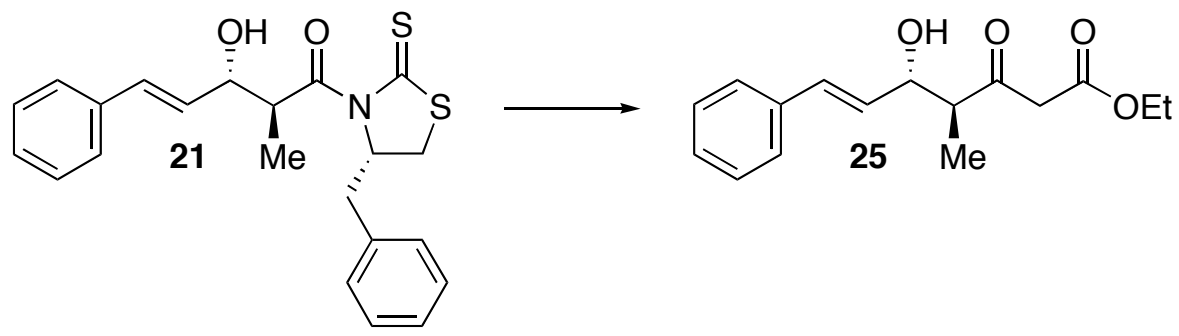

The title compound was prepared according to General Procedure (thiazolidinethione 21, $80.0 \mathrm{mg}, 0.201$ mmol; potassium ethyl malonate, $74.5 \mathrm{mg}, 0.438 \mathrm{mmol} ; \mathrm{MgCl}_{2}, 21.2 \mathrm{mg}, 0.223 \mathrm{mmol}$; imidazole, 13.6 $\mathrm{mg}, 0.200 \mathrm{mmol}$; stirred overnight) and isolated as a pale yellow oil (29.0 $\mathrm{mg}, 52 \%$ yield): ${ }^{1} \mathrm{H}$ NMR $\left(500 \mathrm{MHz}, \mathrm{CDCl}_{3}\right) \delta 7.40-7.26(\mathrm{~m}, 5 \mathrm{H}), 6.63(\mathrm{~d}, 1 \mathrm{H}, J=16 \mathrm{~Hz}), 6.18(\mathrm{dd}, 1 \mathrm{H}, J=7.3,16 \mathrm{~Hz}), 4.38$ (ap t, $1 \mathrm{H}, J=7.8 \mathrm{~Hz}), 4.20(\mathrm{q}, 2 \mathrm{H}, J=6.8 \mathrm{~Hz}), 3.60($ ap d, $2 \mathrm{H}, J=7.8 \mathrm{~Hz}), 2.93$ (ap quintet, $1 \mathrm{H}, J=7.3$ $\mathrm{Hz}), 2.35$ (bs, 1H), $1.28(\mathrm{t}, 3 \mathrm{H}, J=8.2 \mathrm{~Hz}), 1.14(\mathrm{~d}, 3 \mathrm{H}, J=6.8 \mathrm{~Hz}) ;{ }^{13} \mathrm{C} \mathrm{NMR}\left(100 \mathrm{MHz}, \mathrm{CDCl}_{3}\right)$ ठ 136.4, 132.9, 129.4, 128.9, 128.3, 126.8, 75.7, 51.9, 50.1, 14.3, 13.9; IR (film) 3504, 2980, 1741, 1709, 1458, 1368, 1311, 1260, 1156, 1020, 959, 751, $694 \mathrm{~cm}^{-1} ;[\alpha]_{\mathrm{D}}^{25}=+35.3^{\circ}\left(c=1.50, \mathrm{CH}_{2} \mathrm{Cl}_{2}\right)$; HRMS (CI): Exact mass calcd for $\mathrm{C}_{16} \mathrm{H}_{20} \mathrm{O}_{4}+\mathrm{NH}_{4}\left[\mathrm{M}+\mathrm{NH}_{4}\right]^{+}, 294.1706$. Found 294.1705.

\section{Ethyl $(E)(4 R, 5 S)-5-h y d r o x y-4-m e t h y l-3-o x o-7-p h e n y l h e p t-6-e n o a t e ~(23)$}<smiles>CCOC(=O)CC(=O)C(C)[C@H](O)/C=C/c1ccccc1</smiles>

The title compound was prepared according to General Procedure (thiazolidinethione 22, $89.8 \mathrm{mg}, 0.292$ mmol; potassium ethyl malonate, $98.8 \mathrm{mg}, 0.580 \mathrm{mmol} ; \mathrm{MgCl}_{2}, 27.4 \mathrm{mg}, 0.288 \mathrm{mmol}$; imidazole, 20.5 $\mathrm{mg}, 0.301 \mathrm{mmol}$; stirred overnight) and isolated as a pale yellow oil (49.6 mg, 61\% yield). All spectral data were identical to those reported for $\mathbf{2 3}$ above.

General Procedure: Lactonization/Methylation. To a $25 \mathrm{~mL}$ round-bottom flask was added $\delta$-hydroxy- $\beta$-ketoester $(0.656 \mathrm{mmol}, 1$ equiv) in methanol (3.3 mL, $0.2 \mathrm{M}$ in ester). Powdered anhydrous $\mathrm{K}_{2} \mathrm{CO}_{3}$ (1.311 mmol, 2 equiv) was added and the suspension was stirred at rt. After $2 \mathrm{~h}$, TLC indicated complete lactonization. The methanol solvent was removed in vacuo and was replaced with acetone (3.3 $\mathrm{mL}) . \mathrm{Me}_{2} \mathrm{SO}_{4}$ (1.311 mmol, 2 equiv) was added and the suspension was stirred overnight. The reaction was diluted with EtOAc $(20 \mathrm{~mL})$ and was washed with $0.5 \mathrm{M} \mathrm{HCl}(20 \mathrm{~mL})$. The aqueous layer was extracted with EtOAc ( 2 x $3 \mathrm{~mL})$ and the combined organic layers were dried over $\mathrm{Na}_{2} \mathrm{SO}_{4}$ and concentrated in vacuo. Flash chromatography $(0 \rightarrow 50 \%$ EtOAc/hexanes $)$ afforded the pure kavalactone. 

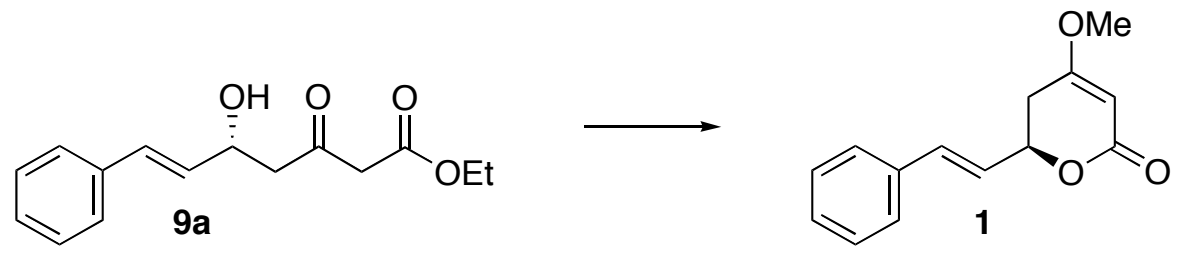

The title compound was prepared according to General Procedure ( $\delta$-hydroxy- $\beta$-ketoester 9a, $172 \mathrm{mg}$, $0.656 \mathrm{mmol} ; \mathrm{K}_{2} \mathrm{CO}_{3}, 181 \mathrm{mg}, 1.311 \mathrm{mmol} ; \mathrm{Me}_{2} \mathrm{SO}_{4}, 124 \mu \mathrm{L}, 1.311 \mathrm{mmol}$; stirred overnight) and isolated as white crystals $\left(131 \mathrm{mg}, 87 \%\right.$ yield): ${ }^{1} \mathrm{H}$ NMR $\left(300 \mathrm{MHz}, \mathrm{CDCl}_{3}\right) \delta$ 7.44-7.24 (m, 5H), $6.74(\mathrm{~d}, 1 \mathrm{H}, \mathrm{J}$ $=16.0 \mathrm{~Hz}), 6.26(\mathrm{dd}, 1 \mathrm{H}, \mathrm{J}=16.0,6.2 \mathrm{~Hz}), 5.20(\mathrm{~s} 1 \mathrm{H}), 5.11-5.02(\mathrm{~m}, 1 \mathrm{H}), 3.77(\mathrm{~s}, 3 \mathrm{H}), 2.67(\mathrm{dd}, 1 \mathrm{H}, \mathrm{J}$ $=17.1,10.7 \mathrm{~Hz}), 2.55(\mathrm{dd}, 1 \mathrm{H}, \mathrm{J}=17.1,4.6 \mathrm{~Hz}) \mathrm{ppm} ;{ }^{13} \mathrm{C} \mathrm{NMR}\left(75 \mathrm{MHz}, \mathrm{CDCl}_{3}\right) \delta 172.7,167.2,136.2$, 133.6, 129.1, 128.8, 127.1, 125.9, 91.0, 76.3, 56.5, 33.7 ppm ; IR (film) 1703, 1626, 1394, 1248, 1233, 1063, 1024, 971, 747, $693 \mathrm{~cm}^{-1}$; m.p. $=112.2-113.0{ }^{\circ} \mathrm{C} ;[\alpha]_{\mathrm{D}}{ }^{20}=+124.3^{\circ}(c=1.00$, EtOH$) ; \mathrm{HRMS}$ (EI): Exact mass calcd for $\mathrm{C}_{14} \mathrm{H}_{14} \mathrm{O}_{3}[\mathrm{M}]^{+}: 230.0943$; Found: 230.0941; Anal calcd for $\mathrm{C}_{14} \mathrm{H}_{14} \mathrm{O}_{3}: \mathrm{C}, 73.03 \%$, $\mathrm{H}, 6.13 \%$; Found: $\mathrm{C}, 72.86 \%, \mathrm{H}, 6.00 \%$. HPLC comparison to a racemic sample indicated an enantiomeric purity of $97.4 \%$ ee (Chiralcel AD column, $7 \%{ }^{i} \mathrm{PrOH} / \mathrm{hexanes,}, 0.7 \mathrm{~mL} / \mathrm{min}, 254 \mathrm{~nm}$ ).

\section{(S)-5,6-dihydro-4-methoxy-6-phenethylpyran-2-one, (+)-7,8-dihydrokavian (2)}
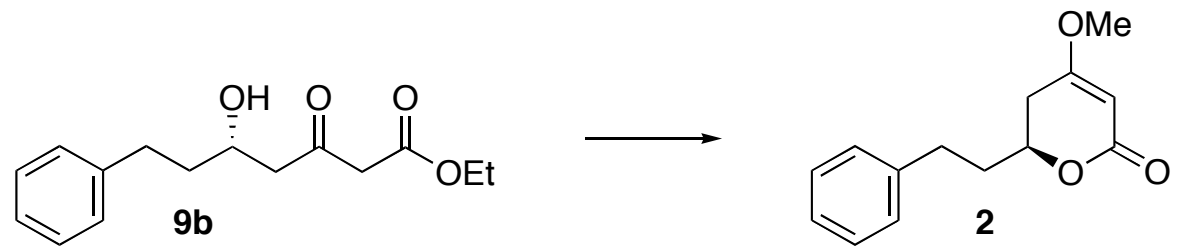

The title compound was prepared according to General Procedure ( $\delta$-hydroxy- $\beta$-ketoester $\mathbf{9 b}, 288 \mathrm{mg}$, $1.09 \mathrm{mmol} ; \mathrm{K}_{2} \mathrm{CO}_{3}, 301 \mathrm{mg}, 2.18 \mathrm{mmol} ; \mathrm{Me}_{2} \mathrm{SO}_{4}, 206 \mu \mathrm{L}, 2.18 \mathrm{mmol}$; stirred overnight) and isolated as off-white crystals (210 mg, 83\% yield): ${ }^{1} \mathrm{H}$ NMR $\left(300 \mathrm{MHz}, \mathrm{CDCl}_{3}\right) \delta 7.32-7.16(\mathrm{~m}, 5 \mathrm{H}), 5.14(\mathrm{~s}, 1 \mathrm{H})$, 4.42-4.30 (m, 1H), $3.72(\mathrm{~s}, 3 \mathrm{H}), 2.94-2.71(\mathrm{~m}, 2 \mathrm{H}), 2.50(\mathrm{dd}, 1 \mathrm{H}, \mathrm{J}=17.0,11.9 \mathrm{~Hz}), 2.30(\mathrm{dd}, 1 \mathrm{H}, \mathrm{J}=$ 17.0, $3.9 \mathrm{~Hz}), 2.20-2.05(\mathrm{~m}, 1 \mathrm{H}), 1.99-1.85(\mathrm{~m}, 1 \mathrm{H}) \mathrm{ppm} ;{ }^{13} \mathrm{C}$ NMR $\left(75 \mathrm{MHz}, \mathrm{CDCl}_{3}\right) \delta 172.7,167.2$, 140.8, 128.5, 128.4, 126.1, 90.3, 74.7, 56.0, 36.3, 33.0, 30.9 ppm; IR (KCl wafer) 3026, 2941, 2856, 1707, 1624, 1497, 1454, 1443, 1396, 1374, 1294, 1223, 1090, 1038, 997, 869, 823, 751, $701 \mathrm{~cm}^{-1}$; m.p. $=57.3-58.0{ }^{\circ} \mathrm{C} ;[\alpha]_{\mathrm{D}}{ }^{25}=+31.1^{\circ}(c=1.00, \mathrm{MeOH})$; HRMS (EI): Exact mass calcd for $\mathrm{C}_{14} \mathrm{H}_{16} \mathrm{O}_{3}[\mathrm{M}]^{+}$: 232.1099; Found: 232.1107; Anal calcd for $\mathrm{C}_{14} \mathrm{H}_{16} \mathrm{O}_{3}$ : C, 72.39\%, H, 6.94\%; Found: C, 72.18\%, H, $6.93 \%$.

(R)-6-((E)-2-(tributylstannyl)vinyl)-5,6-dihydro-4-methoxypyran-2-one (11)<smiles>CCOC(=O)CC(=O)C[C@@H](O)/C=C/[SnH2]C(C)(C)C</smiles><smiles>CCC(C)[SnH2]/C=C/C1CC(OC)=CC(=O)O1</smiles> 
The title compound was prepared according to General Procedure ( $\delta$-hydroxy- $\beta$-ketoester 9c, $64 \mathrm{mg}$, $0.135 \mathrm{mmol} ; \mathrm{K}_{2} \mathrm{CO}_{3}, 37 \mathrm{mg}, 0.268 \mathrm{mmol} ; \mathrm{Me}_{2} \mathrm{SO}_{4}, 25 \mu \mathrm{L}, 0.268 \mathrm{mmol}$; stirred overnight) and isolated as an orange oil (47 mg, 79\% yield): ${ }^{1} \mathrm{H}$ NMR (300 MHz, $\left.\mathrm{CDCl}_{3}\right) \delta 6.37(\mathrm{~d}, 1 \mathrm{H}, \mathrm{J}=19.2 \mathrm{~Hz}), 6.06(\mathrm{dd}$, $1 \mathrm{H}, \mathrm{J}=19.2,5.1 \mathrm{~Hz}), 5.15(\mathrm{~s}, 1 \mathrm{H}), 4.91-4.80(\mathrm{~m}, 1 \mathrm{H}), 3.75(\mathrm{~s}, 3 \mathrm{H}), 2.58(\mathrm{dd}, \mathrm{J}=17.1,10.3 \mathrm{~Hz}), 2.48$ $(\mathrm{dd}, \mathrm{J}=17.1,5.0 \mathrm{~Hz}), 1.57-1.42(\mathrm{~m}, 6 \mathrm{H}), 1.39-1.23(\mathrm{~m}, 6 \mathrm{H}), 0.97-0.84(\mathrm{~m}, 15 \mathrm{H}) \mathrm{ppm} ;{ }^{13} \mathrm{C}$ NMR $(75$ $\left.\mathrm{MHz}, \mathrm{CDCl}_{3}\right) \delta 172.35,166.95,143.51,132.95,90.51,77.91,55.99,32.81,28.98,27.20,13.65,9.47$ ppm ; IR (film) 2956, 2926, 2871, 2853, 1715, 1627, 1463, 1382, 1279, 1227, 1065, 1023, 995, 960, $873,824,691,667 \mathrm{~cm}^{-1} ;[\alpha]_{\mathrm{D}}{ }^{21.5}=+47.8^{\circ}\left(c=1.00, \mathrm{CH}_{2} \mathrm{Cl}_{2}\right)$; Anal calcd for $\mathrm{C}_{20} \mathrm{H}_{36} \mathrm{O}_{3} \mathrm{Sn}: \mathrm{C}, 54.20 \%, \mathrm{H}$, 8.19\%; Found: C, 54.29\%, H, 8.28\%.

\section{5,6-dihydro-4-methoxy-6-styrylpyran-2-one, $( \pm)$-kavain $(( \pm)-1)$}
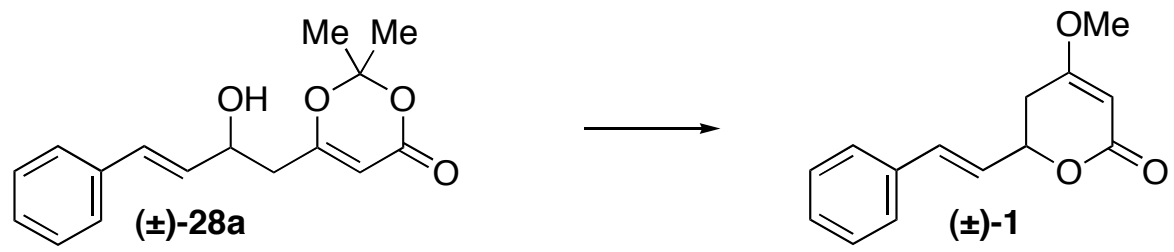

The title compound was prepared according to General Procedure ( $\delta$-hydroxyester $( \pm)-28 a, 157 \mathrm{mg}$, $0.572 \mathrm{mmol} ; \mathrm{K}_{2} \mathrm{CO}_{3}, 158 \mathrm{mg}, 1.145 \mathrm{mmol} ; \mathrm{Me}_{2} \mathrm{SO}_{4}, 108 \mu \mathrm{L}, 1.145 \mathrm{mmol}$; stirred overnight) and isolated from as colorless crystals ( $94 \mathrm{mg}, 71 \%$ yield): All spectral data were identical to those reported for 1 above except for the melting point $\left(145.8-146.9{ }^{\circ} \mathrm{C}\right)$ and absence of optical activity.

\section{5,6-dihydro-4-methoxy-6-phenethylpyran-2-one, ( $( \pm)-7,8$-dihydrokavian $(( \pm)-2)$}

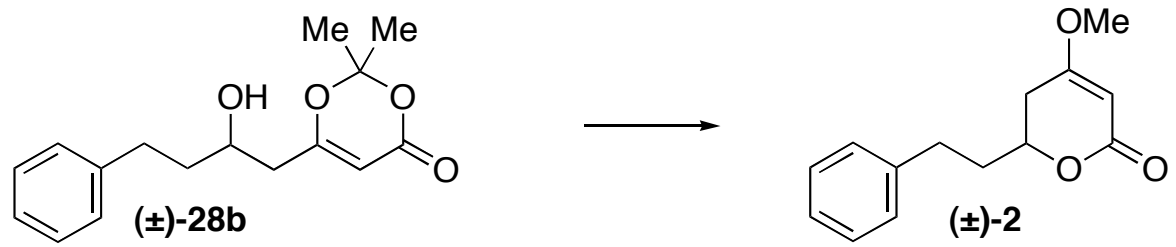

The title compound was prepared according to General Procedure ( $\delta$-hydroxyester ( $\mathbf{\pm})-\mathbf{2 8 b}, 206 \mathrm{mg}$, $0.746 \mathrm{mmol} ; \mathrm{K}_{2} \mathrm{CO}_{3}, 206 \mathrm{mg}, 1.491 \mathrm{mmol} ; \mathrm{Me}_{2} \mathrm{SO}_{4}, 141 \mu \mathrm{L}, 1.491 \mathrm{mmol}$; stirred overnight) and isolated as colorless crystals (155 mg, 90\% yield): All spectral data were identical to those reported for 2 above except for absence of optical activity.

General Procedure: Stille Coupling. To a $10 \mathrm{~mL}$ round-bottom flask was sequentially added the aryl iodide $(0.236 \mathrm{mmol}, 1.1$ equiv), THF or toluene $(1.0 \mathrm{~mL})$, the phosphine ligand $(0.0189 \mathrm{mmol}, 0.088$ equiv), and $\mathrm{Pd}_{2}(\mathrm{dba})_{3}(0.0047,0.022$ equiv). After stirring at $\mathrm{rt}$ for $15 \mathrm{~min}$, the solution was warmed to $50{ }^{\circ} \mathrm{C}$, stannane $11(0.214 \mathrm{mmol}, 1.0$ equiv) was added in THF $(0.5 \mathrm{~mL})$, and the reaction was stirred overnight. The solvent was removed in vacuo and the brown residue was subjected to flash chromatography $(0 \rightarrow 50 \%$ EtOAc/hexanes). Stirring with triamine-functionalized silica gel (SiliCycle: $\mathrm{Si}$-Triamine) removed the residual $\mathrm{Bu}_{3} \mathrm{SnI}$ to afford the pure kavalactones. 


\section{(R)-5,6-dihydro-4-methoxy-6-styrylpyran-2-one, (+)-kavain (1)}

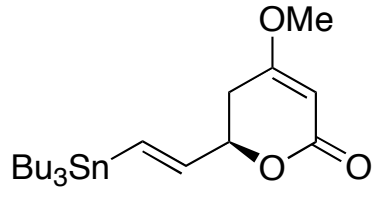

11

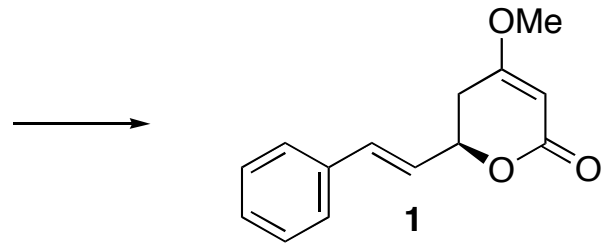

The title compound was prepared according to General Procedure (iodobenzene, $26.3 \mu \mathrm{L}, 0.236 \mathrm{mmol}$; $\mathrm{P}(2 \text {-furyl })_{3}, 4.4 \mathrm{mg}, 0.0189 \mathrm{mmol} ; \mathrm{Pd}_{2}(\mathrm{dba})_{3}, 4.3 \mathrm{mg}, 0.0047 \mathrm{mmol}$, stannane 11, $95 \mathrm{mg}, 0.214 \mathrm{mmol}$; stirred overnight) and isolated as white crystals (38 $\mathrm{mg}, 75 \%$ yield): All spectral data were identical to those reported for $\mathbf{1}$ above.

(R)-6-(4-methoxystyryl)-5,6-dihydro-4-methoxypyran-2-one), (+)-5,6-dihydroyangonin (12)
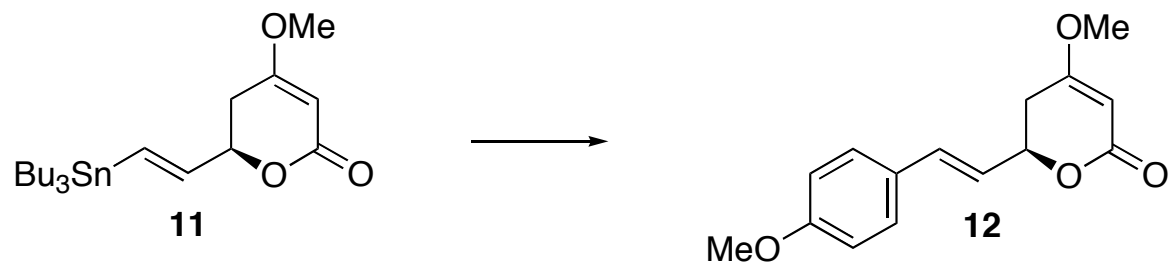

The title compound was prepared according to General Procedure (4-iodoanisole, $55 \mathrm{mg}, 0.235 \mathrm{mmol}$; $\mathrm{P}(o-\mathrm{MeO}-\mathrm{Ph})_{3}, 3.6 \mathrm{mg}, 0.0103 \mathrm{mmol} ; \mathrm{Pd}_{2}(\mathrm{dba})_{3}, 2.4 \mathrm{mg}, 0.0026 \mathrm{mmol}$, stannane 11, $52 \mathrm{mg}, 0.117$ mmol; stirred overnight) and isolated as colorless crystals (16 mg, $52 \%$ yield): ${ }^{1} \mathrm{H} \mathrm{NMR}(500 \mathrm{MHz}$, $\left.\mathrm{CDCl}_{3}\right) \delta 7.32(\mathrm{~d}, 2 \mathrm{H}, \mathrm{J}=8.7 \mathrm{~Hz}), 6.86(\mathrm{~d}, 2 \mathrm{H}, \mathrm{J}=8.7 \mathrm{~Hz}), 6.67(\mathrm{~d}, 1 \mathrm{H}, \mathrm{J}=15.9 \mathrm{~Hz}), 6.12(\mathrm{dd}, 1 \mathrm{H}, \mathrm{J}=$ $15.9,6.5 \mathrm{~Hz}), 5.19(\mathrm{~s}, 1 \mathrm{H}), 5.06-4.99(\mathrm{~m}, 1 \mathrm{H}), 3.81(\mathrm{~s}, 3 \mathrm{H}), 3.76(\mathrm{~s}, 3 \mathrm{H}), 2.65(\mathrm{dd}, 1 \mathrm{H}, \mathrm{J}=17.1,10.9$ $\mathrm{Hz}), 2.53(\mathrm{dd}, 1 \mathrm{H}, \mathrm{J}=17.1,4.3 \mathrm{~Hz}) \mathrm{ppm} ;{ }^{13} \mathrm{C} \mathrm{NMR}\left(125 \mathrm{MHz}, \mathrm{CDCl}_{3}\right) \delta 172.3,166.8,159.7,132.8$, 128.4, 127.9, 123.2, 114.0, 90.5, 76.1, 56.0, 55.3, 33.4 ppm ; IR (film) 1705, 1623, 1512, 1389, 1249, $1224,1177,1016,816 \mathrm{~cm}^{-1} ;[\alpha]_{\mathrm{D}}^{19}=+99.8^{\circ}\left(c=1.00, \mathrm{CH}_{2} \mathrm{Cl}_{2}\right)$.

$(R)-6-((E)-2-(b e n z o[d][1,3]$ dioxol-6-yl)vinyl)-5,6-dihydro-4-methoxypyran-2-one, (+)-methysticin (3)

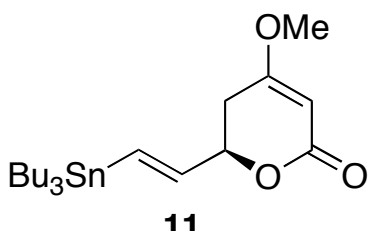

11

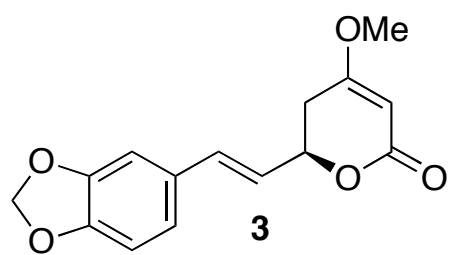

The title compound was prepared according to General Procedure (1-iodo-3,4-methylenedioxybenzene $73 \mathrm{mg}, 0.293 \mathrm{mmol} ; \mathrm{P}(o-\mathrm{MeO}-\mathrm{Ph})_{3}, 4.5 \mathrm{mg}, 0.0129$ mmol; $\mathrm{Pd}_{2}(\mathrm{dba})_{3}, 2.9 \mathrm{mg}, 0.0032 \mathrm{mmol}$, stannane 11, $65 \mathrm{mg}, 0.147 \mathrm{mmol}$; stirred overnight) and isolated as off-white crystals $\left(18 \mathrm{mg}, 44 \%\right.$ yield): ${ }^{1} \mathrm{H}$ NMR $\left(500 \mathrm{MHz}, \mathrm{CDCl}_{3}\right) \delta 6.92(\mathrm{~d}, 1 \mathrm{H}, \mathrm{J}=1.6 \mathrm{~Hz}), 6.83(\mathrm{dd}, 1 \mathrm{H}, \mathrm{J}=8.0,1.6 \mathrm{~Hz}), 6.76(\mathrm{~d}, 1 \mathrm{H}, \mathrm{J}=8.0$ $\mathrm{Hz}), 6.38(\mathrm{~d}, 1 \mathrm{H}, \mathrm{J}=15.9 \mathrm{~Hz}), 6.09$ (dd, 1H, J = 15.9, $6.4 \mathrm{~Hz}), 5.96(\mathrm{~s}, 2 \mathrm{H}), 5.19(\mathrm{~s}, 1 \mathrm{H}), 5.05-4.99$ (m, 1H), 3.77 (s, 3H), $2.65(\mathrm{dd}, 1 \mathrm{H}, \mathrm{J}=17.1,10.9 \mathrm{~Hz}), 2.53(\mathrm{dd}, 1 \mathrm{H}, \mathrm{J}=17.1,4.3 \mathrm{~Hz}) \mathrm{ppm} ;{ }^{13} \mathrm{C}$ NMR $(125$ $\left.\mathrm{MHz}_{\mathrm{CDCl}}\right) \delta 172.3,166.7,148.1,147.8,132.9,130.2,123.6,121.7,108.3,105.8,101.2,90.6,76.0$, 
56.1, 33.4 ppm ; IR (film) 1705, 1623, 1505, 1490, 1446, 1392, 1250, 1223, 1096, 1036, 965, 930, 866, $798 \mathrm{~cm}^{-1} ;[\alpha]_{\mathrm{D}}^{19}=+119.5^{\circ}\left(c=1.00, \mathrm{CH}_{2} \mathrm{Cl}_{2}\right)$. 